\title{
EXISTENCE OF THE LEAST AND THE GREATEST ELEMENTS OF A SUBSET OF THE LINDENBAUM ALGEBRA
}

\author{
By \\ Mitio TAKano
}

\section{Introduction.}

The purpose of this note is to answer the following problem.

Problem. Let $P$ be a propositional letter and $G(P)$ a formula. Does the set $\{A \mid \vdash G(A)\}$ always have the least and the greatest elements unless it is empty?

To be accurate, of course, the above problem must be understood in the Lindenbaum algebra.

The answer is "no" if $\vdash$ denotes the provability in the intuitionistic logic (§1), while "yes" if the classical logic is concerned with (§2).

The author expresses his thanks to Dr. Masazumi Hanazawa, who raised him the above problem.

\section{§1. The intuitionistic case.}

In this section we understand by $\vdash$ the provability in the intuitionistic logic. Then the problem is negative since Proposition 1 below shows an example of a non-empty set which has neither the least nor the greatest element.

Proposition 1. Let $Q$ and $R$ be distinct propositional letters and $G(P)$ be the formula $[P \sim Q] \vee[P \sim R]$.

$\left.1^{\circ}\right) \vdash G(Q)$ and $\vdash G(R)$.

$\left.2^{\circ}\right)$ There is no formula $B$ such that $(\mathrm{a}) \vdash G(B)$ and $(\mathrm{b})$ if $\vdash G(A)$ then $\vdash B \supset A$ for every $A$.

$\left.3^{\circ}\right)$ There is no formula $B$ such that (a) $\vdash G(B)$ and $\left(\mathrm{b}^{\prime}\right)$ if $\vdash G(A)$ then $\vdash A \supset B$ for every $A$.

ProOF. $\left.1^{\circ}\right)$ is clear.

$2^{\circ}$ ) We assume, on the contrary to the conclusion, that any formula $B$ Recevied March 25, 1985. 
satisfying (a) and (b) is given. By $1^{\circ}$ ) and (b) we have $\vdash B \supset Q$ and $\vdash B \supset R$, from which together with (a) follows $\vdash[Q \supset R] \vee[R \supset Q]$ which is absurd. Hence no such $B$ exists.

$3^{\circ}$ ) is proved similarly.

\section{§2. The classical case.}

In this section we understand by $\vdash$ the provability in the classical logic. Then we see by Proposition 2 below that the set $\{A \mid 1-G(A)\}$ has the least element $\neg G(\boldsymbol{F})$ and the greatest element $G(\boldsymbol{T})$ unless it is empty, where for brevity's sake we assume that the propositional constants $\boldsymbol{T}$ (truth) and $\boldsymbol{F}$ (falsity) are admitted. So the problem stated in the introduction is affirmative.

We remark that Proposition $2,4^{\circ}$ ) has, essentially, already been obtained in Maehara [1, Appendix $]$.

Proposition 2. Let be given a propositional letter $P$ and a formula $G(P)$.

$\left.1^{\circ}\right) \vdash G(A) \supset[\neg G(\boldsymbol{F}) \supset A]$.

$\left.2^{\circ}\right) \vdash G(A) \supset G(\neg G(\boldsymbol{F}))$.

$\left.3^{\circ}\right) \vdash G(A) \supset[A \supset G(\boldsymbol{T})]$.

$\left.4^{\circ}\right) \vdash G(A) \supset G(G(\boldsymbol{T}))$.

PROOF. We first remark the facts that

$$
\vdash A \supset[A \sim \boldsymbol{T}], \quad \vdash \neg A \supset[A \sim \boldsymbol{F}], \quad \vdash[A \sim B] \supset[G(A) \sim G(B)],
$$

and so

$$
\vdash A \supset[G(A) \sim G(\boldsymbol{T})], \quad \vdash \neg A \supset[G(A) \sim G(\boldsymbol{F})], \quad \vdash G(\boldsymbol{T}) \wedge G(\boldsymbol{F}) \supset G(A) .
$$

$\left.1^{\circ}\right)$ follows from $\vdash \neg A \supset[G(A) \sim G(\boldsymbol{F})]$.

$2^{\circ}$ ) From $\vdash \neg \neg G(\boldsymbol{F}) \supset[G(\neg G(\boldsymbol{F})) \sim G(\boldsymbol{F})]$ it follows $\vdash A \wedge G(\boldsymbol{F}) \supset[G(A) \supset$ $G(\neg G(\boldsymbol{F}))]$, while from $\left.1^{\circ}\right)$ it follows $\vdash \neg A \wedge \neg G(\boldsymbol{F}) \supset[G(A) \supset G(\neg G(\boldsymbol{F}))]$; so $\vdash \neg[A \sim \neg G(\boldsymbol{F})] \supset[G(A) \supset G(\neg G(\boldsymbol{F}))]$, from which together with $\vdash[A \sim \neg G(\boldsymbol{F})]$ $\supset[G(A) \sim G(\neg G(\boldsymbol{F}))]$ follows $\left.2^{\circ}\right)$.

$\left.3^{\circ}\right)$ follows from $\vdash A \supset[G(A) \sim G(T)]$.

$\left.4^{\circ}\right)$ From $\vdash \neg A \supset[G(A) \sim G(\boldsymbol{F})]$ and $\vdash G(\boldsymbol{T}) \wedge G(\boldsymbol{F}) \supset G(G(\boldsymbol{T}))$ it follows $\vdash \neg A \wedge G(\boldsymbol{T}) \supset[G(A) \supset G(G(\boldsymbol{T}))]$, while from $\left.3^{\circ}\right)$ it follows $\vdash A \wedge \neg G(\boldsymbol{T}) \supset[G(A) \supset$ $G(G(\boldsymbol{T}))]$; so $\vdash \neg[A \sim G(\boldsymbol{T})] \supset[G(A) \supset G(G(\boldsymbol{T}))]$, from which together with $\vdash[A \sim G(\boldsymbol{T})] \supset[G(A) \sim G(G(\boldsymbol{T}))]$ follows $\left.4^{\circ}\right)$.

Next, consider the following modified problem.

Problem. Let $P$ be an n-ary predicate letter and $G(P)$ a formula. Does the set 


$$
\left\{\lambda x_{1} \cdots x_{n} A\left(x_{1}, \cdots, x_{n}\right) \mid \vdash G\left(\lambda x_{1} \cdots x_{n} A\left(x_{1}, \cdots, x_{n}\right)\right)\right\}
$$

always have the least and the greatest elements unless it is empty?

The answer is "no" as the following proposition shows. The proof is similar to that of Proposition 1 since it is not the case that $\vdash \forall x[Q x \supset R x] \vee \forall x[R x \supset Q x]$.

PROPOSITION 3. Let $Q$ and $R$ be distinct unary predicate letters and $G(P)$ be the formula $\forall x[P x \sim Q x] \vee \forall x[P x \sim R x]$.

$\left.1^{\circ}\right) \vdash G(\lambda x Q x)$ and $\vdash G(\lambda x R x)$.

$\left.2^{\circ}\right)$ There is no $\lambda x B(x)$ such that $(\mathrm{a}) \vdash G(\lambda x B(x))$ and $(\mathrm{b})$ if $\vdash G(\lambda x A(x))$ then $\vdash \forall x[B(x) \supset A(x)]$ for every $\lambda x A(x)$.

$\left.3^{\circ}\right)$ There is no $\lambda x B(x)$ such that (a) $\vdash G(\lambda x B(x))$ and $\left(\mathrm{b}^{\prime}\right)$ if $\vdash G(\lambda x A(x))$ then $\vdash \forall x[A(x) \supset B(x)]$ for every $\lambda x A(x)$.

\title{
Reference
}

[1] Maehara, S., The predicate calculus with $\varepsilon$-symbol, J. Math. Soc. Japan 7 (1955), 323-344.

\author{
Department of Mathematics \\ Faculty of Education \\ Niigata University \\ Niigata, 950-21 Japan
}

